\title{
61
}

\section{Formulating a Successful Management Strategy}

\author{
Rick STURM, US West Advanced Technologies, U.S.A.
}

"Rightsizing" is often used as a euphemism for work force reductions. In network management, "rightsizing" is not limited to the question of how an organization can do its work with fewer people. At the same time that they are being pressured to minimize the size of their organization, managers are being asked to significantly increase the scope of their responsibilities to include such things as the management of distributed systems. This presentation will address the factors that are essential in formulating a successful strategy to respond to these conflicting demands. 